not pass the pores of this filter, at least in sufficient quantity to induce infection.

A sheep which had its nostrils swabbed with an emulsion of the brain and spinal cord from an infected monkey showed no after-effects.

\section{Other Animals.}

We failed to convey the disease to four dogs, two kittens, three rabbits, two guinea-pigs, and one fowl.

\section{SUMmarT.}

1. Clinically.-An account of a form of encephalo. myelitis, occurring as an epidemic in the late summer of 1917 and 1918, confined almost entirely to certain dry inland parts of New South Wales; appearing as an acute and severe illness, often abrupt in onset, and fatal in 70 per cent. of cases; affecting children mainly but not sparing adults ; characterized by general signs of cerebro-spinal irritation-namely, convulsions, rigidity, heightened reflex excitability, mental obfuscation, and loss of consciousness ; accompanied by fever and gastric and other disturbances; terminating in exhaustion, coma, and death in a few days, or in rapid recovery, usually complete, but in a few instances attended by flaccid paralysis or mental disorder.

2. Histologically.-Exhaustive microscopic examination of the nervous system in 16 cases showed acute encephalo. myelitis, universally distributed ; characterized by vascular engorgement, "sleeving" of veins with lymphocyte-like cells, isolated "colonies" in and infiltration of the tissues with similar cells, and a tendency to capillary haemorrhage; affecting grey substance more than white on account of the difference in vascular supply; and seldom grossly destructive as in " acute poliomyelitis."

3. Experimentally.-By intracerebral inoculation of an emulsion of portions of the parent nervous system the diseage has been conveyed from human subject to monkey (Macacus rhesus); from monkey to monkey, in series; from monkey to sheep; from sheep to sheep; back from sheep to monkey; and on again through monkeys; and lastly, from monkey to one horse and one calf-all of which results were confirmed by histological examination, showing changes unequivocally corresponding with those found in homo.

4. The disease in monkeys was eminently fatal, and was mainly characterized by weakness, drowsiness, and intense inco-ordination of movement. Flaccid paralysis was not a dominant feature. Some sheep gave no response to inoculation, others showed slight or no signs of disease, proving a certain degree of immunity. In the "positive" sheep convulsions commonly caused death. The inoculated horse and culf also died from convulsions.

\section{Conchusion.}

The disease, from the anatomical standpoint, may be called "acute encephalo-myelitis."

It resembles "acute poliomyelitis" and "encephalitis lethargica" inasmuch as the responsible, undiscovered virus has an evident affinity for the central nervous system; probably requires incubation therein, under favourable conditions, to perfect its nocive power, and produces histological changes which follow the blood vessels, hence suggesting that all three diseases may be due to mutants of the one virus.

On the contrary, however, the individuality of this epidemic; the preponderance of signs of cerebro-spinal irritation; the high rate of mortality; the common affection of adults; the infrequency of paralysis of cranial or peripheral nerves; the widespread changes in the nervous system without gross focal lesions; the susceptibility of the sheep, horse, and calf, as well as the monkey and the reproduction of preponderating signs of cerebral irritation and of the human histological changes in all these animals, favour the conclusion that the disease is a distinot entity.

AT the last meeting of the Zoological Society of London Mr. E. G. Boulenger, F.Z.S., exhibited a series of living specimens of the British rats and their varieties, and stated that during the past four years there was evidence that the so-called old 'English black rat had increased in numbers. Lieut.-Colonel S. Monckton Copeman, F.R.S., F.ż.S., read a paperion ex periments on sexx deter'mination, illustrating his remarks by a series of cherts.

\section{TWO OUTBREAKS OF MILD DYSENTERY ASSOCIATED WITH THE}

SCHMITZ BACILLUS (B, AMBIGUUS ANDREWES), BY

MAJOR W. BROUGHTON-ALCOCK, R.A.M.C.(S.R.) No. - General Hosprtar, I.E.F.

Some clinical and laboratory observations made during the investigations of two outbreaks of a mild form of dysentery during July, 1918, are given in this note. They appear of particular interest from the fact that the labora. tory work carried out indicated that the causal organism was the $B$. dysenteriae Schmitz (B. ambiguus Andrewes), and that the first outbreak was of rare extent.

In Professor Andrewes's account ${ }^{1}$ of his investigations into several strains of bacillary organisms, which he has included in the general term "dysentery group," are detailed his findings when working upon a similar micro. organism, together with some interesting remarks on what have been frequently termed "atypical " dysentery bacilli.

A bacillus of similar biological characters has been isolated from dysenteric stools by several workers in various countries. It has been my previous experience while working in the Mediterranean littoral to isolate it very rarely, and then from the stool of a patient suffering with a mild form of dysentery.

Clinically the cases to be described presented signs and symptoms which resembled those seen in mild cases due to the Flexner.Hiss bacillus. The more severe form of dysentery and the associated toxicity so generally found when the infective micro-organism is the Shiga bacillus were not observed in any case.

The first outbreak was amongst troops belonging to one camp. Those in an adjoining camp remained free from infection. The troops in the camp affected had recently arrived from a distant allied country, while in the other they were British, and had been in Italy for several months. Within the period of one week 12 officers and 350 men, out of a total of 3,000 troops, fell ill with a com. paratively mild form of dysentery associated with vomiting and abdominal pain. The onset was sudden and acute. In many there was collapse, and the temperature rose to around $102^{\circ} \mathrm{F}$. The duration of the attack averaged three to four days, when the patient was practically able to return to his duties and the stools were normal in consistency and character. There were no deaths.

The stools were numerous during the first twenty. four to forty-eight hours, and frequency persisted excep. tionally to five or six days. The stools consisted mainly of mucus and bile-stained watery faecal matter; some con. tained blood flakes or cells only, but no clots. The mucus in a few was clear and in plaques, while in others it was nuore opaque and occurred as fine flakes, of colour and consistency varying between clear and white according as the pus and epithelial cells therein were few or numerous.

Microscopical examination of stools showed-save one which contained Lamblia cysts due to a previous infection-an absence of animal paragites an also of vibrios. The minero absence prent in lying packed in the mucus, (2) a motile bacillus of varying length.

Cultures of Stools.-Special .methods were undertaken to determine if culturable vibrios were present, but none were found.

Washed mucus of those stools examined during the first dav or two of symptoms showed comparable cultural results when plated on MacConkey media. The predominant colonies resembled those of the Flexner-Hiss bacillus. They proved $t a$ be of $B$. dysenteriae Schmitz, a non-lactose fermenting short bacillus, which was found to be non-motile, Gram-negative, and to ferment glucose (without gas formation) and not mannite saccharoso, maltose, dulcite, nor lactose. Gelatin was not liquefied. There was no marked change in the litmus milk as prepared. Fresh milk could not be obtained. The ind reaction was positive in twour the reaction was positive in twenty-four to forty-eight hours. The bacillus was not agglutinated

B. pyocyaneus was also isolated, but in a much lesser number of colonies from a small proportion of the same stools.

The second and much smaller outbreak occurred amongst the personnel of a military hospital and at a later date. There was a number of similir cases of mild dysentery, accompanied in some with vomiting, and in the cases investigated the $B$, pyotyneus was not found. 
but the Schmitz bacillus again grew in a considerable number of colonies after plating out the stools:

The Schmitz bacillus was not found when some stools from the above cases were examined at. a later date and when normed in character. No further cases of dysentery oecurred amongst the troops in the same camp or hospital.

The blood serums of seven of the first.series of patients taken nine and fifteen days after the onset of symptoms were tested for agglutinability of the Schmitz bacillus and the $B$. pyjocyaneus isolated with negative results. Similar findings by the agglutination test on the Schmitz bacillus with the serums from cases at the hospital. were obtained. Three strains isolated were submitted to the Michaelis aoid test, but after two hours the result was negative in all tubes; after twenty-four hours' standing fine flocculi were obtained in tube No. 6 .

It appears that these outbreaks were due to an intestinal intoxication dependent upon the proliferation and inild toxicity of the Schmitz bacillus, and that the pathological condition of the intestine present in more severe forms of bacillary dysentery did not develop. Intestinal disorder, often classed as physiological disturbance due to change of climate and of diet, is' not infrequent amongst troops. when they are transferred to a country and to conditions new to them, especially during summer months if that country be subtropical or tropical. But the clinical symptoms and signs observed differ from those seen in such a physiological state, and the direct microscopic and cultural results from stool examinations, which showed considerable numbers of the Schmitz bacillus during the time dysenteric stools were passed and none when the stools returned to normal, are such as lead me to accuse this micro-organism of playing some part in these outbreakg, if it be not the real causal agent. Moveover; I have not yot found the Sohmitz bacillus in a stool from a patient without a history of dysentery, nor in normal stools.

The troops were allowed to eat raw fruit, and the water in the camp affected was at that period not chlorinated; in the adjoining camp it was. The sanitation officer did not trace the cause of the disease to these sources. The day preceding the second outbreak raw tomatoes had been bought and eaten by many of those who became ill. A similar clinical condition arose amongst troops in Malta after eating raw tomatoes in the summer of 1916.

The micro-organism above described has similar biological characters to the $B$. ambiguns described by Andrewes; his strains gave negative results when small or large doses of aërobic broth culture were injected into a rabbit's vein. Its morphological cultural and fermenta. tive characters are similar to those described by Schmitz for his bacillus in an account of an outbreak of dysentery amongst Rumanian prisoners of war, but the symptoms it produced in man were much less severe, and it did not kill the rabbit as did the bacillus isolated by Schmitz. ${ }^{2}$

The extensive and valuable work of Captain Murray ${ }^{3}$ has shown that the bacilli described by Schmitz and Andrewes may be placed in one group, and it will be interesting to learn the comparative results with para-Shiga strains of Dudgeon and others from the Mediterranean littoral.

I beg to thank Lieut.-Colonel J. W. West, C.M.G., R.A.M.C., for permission to publish this article.

${ }^{1}$ Lancet, April 20th, 1918. 2 Zeitsch. f. Hyg., 84, 1917. 3 Journal of the I Lancet, Apris.
R.A.M.G., 1918 .

\section{ANAESTHETICS IN A BRITISH HOSPITAL WITH 'THE SERBIAN ARMY.}

\author{
J. P. GOOD, M,Di, LATt MiJOR R.A.M.C., \\ Anaesthetist, No. - General hospital.
}

Thrs series of anaesthetics was commenced in August 1916, and continued until January, 1918. All the opera. tions took place under canvas in the heart of a country with a subtropical climate. The nationality of the subjects treated was mainly Serbian. The Serbian soldiers were very fit and.strong; men between the ages of 18 and 50 , most of whom had faced the hardship of war, not only during the present struggle, but in the previous Balkan campaigns, and practically all had led an outdoor life. The outstanding features from an anaesthetist's point of view were their good physique, the sound state of their teetl and the absence of dentures; a large percentage did not smoke at all, and those that did were almost all cigarette smokers. A noticeable feature in the early stages was the fear of an anaesthetic. Special account had to be taken of endemic disease (malaria); and occasionally of the effects of dysentery and scurvy in their relation to cardiac complications.

In the first series many of the wounded were in a state of exhaustion on admission, owing to difficulties' of trans port. Where possible, these cases were allowed to rest for some hours before operation.

The methods of administration adopted were as follows :

1. Open administration of a mixture (ether 2 parts, chloroform 1 part), with and. without previous chloroform 1 part), with

2. MoCardie's mitigated ether method with open $\mathrm{E}_{2} \mathrm{C}_{\mathrm{C}}$ sequence (with and without previous administration of morphine and atropine).

3. Closed ether; sometimes with oxygen.

4. Nitrous oxide.

5. Nitrous oxide $\div$ ether sequence.

6. Nitrous oxide with oxygen (improvised)

7. Chloroform (Junker's bottle)

8. Combination of methods $1,2,3$, and 6 with local anaesthesia.

Methods 1 and 2 were the most froquently employed; they were given in a series of cases, with and without previous alka. loidal administration, and the resulta: were compared. The cases which had not had a previous injection of alkaloids were in general head cases and thoracic injuries. The administration of $\mathrm{E}_{2} \mathrm{C}_{1}$ mixture was sometimes preceded by $\frac{1}{2}$ to 1 drachm of chloroform given drop by drop on a Schimmelbusch frame covered with 12-ply gauze and a layer of lint (with a.small.hole the size of half a crown in the centre) over all. In this method the morphine when given was in doses of $t$ or 1 grain one hour to one hour and a half before operation, as nearly as it could be gauged. At this stage no atropine was given.

The anaesthesia obtained was as a whole very satisfactory. Induction was rather slow (about six to eight minutes on the arerage), and in cases newly admitted from the trenches it was often accompanied by great excitement and struggling, whether, morphine had been given or not. The maintenance of anaesthesia was as a rule even and regular; there was little vomiting, and no respiratory disturbance or salivation. It was noted that those patients who liad morphine took a little less anaes. thetic, and generally the induction was quieter and a little quicker. Signs of shock on the table were absent, except in a few cases where the operation had been prolonged and severe, or where haemorrhage had occurred. The period of recovery was quiescent.

\section{1. $E_{2} C_{1}-S c h i m m e l b u s c h$ Frame.}

The following summary shows the quantities of anaes. thetic : used.

(a) With preliminary morphine: 333 operations; average length of operation 37.9 minutes:; average quantity per
operation 11.3 drachms; or; taking an operation of thirty minutes' duration, 8.7 drachrns.

(b) Without preliminary morphine : 231 operations ; average length of operation 33.1 minutes; average quantity per operation 10.6 drachms ; or, taking an operation of thirty minutes' duration, 9.6 drachms.

It will be noted that there is not a big difference in the quantities used in the two classes, the cause being chiefly absence of the ideal conditions for complete success with preliminary alkaloids, such as quietness of surroundings, subdued light, and smooth transport from ward tents to theatre. However, the other benefits were obtained. With regard to the quantities used as compared with open ether administration, the figures given by Silk ${ }^{1}$ and others are 6 to 8 ounces an hour, or even 8 to 10 ounces an hour in Malta.

\section{McCardie's Mitigated Ether.}

In this procedure a Clover apparatus is used into which $1 \mathrm{oz}$. of ether, with half a drachm of chloroform, are placed in the container, and 21 minims of $\mathrm{E}_{2} \mathrm{C}_{1}$ mixture in the bag. The facepiece is closely and firmly applied; induction is commenced by rebreathing from the bag only for forty to sixty seconds, and followed by turning on the mitigated ether. Watch is kept for cyanosis, and if this appears (which is rare), one or two breaths of air are given. Induction was almost always easy, quiet, and rapid, taking four or five- minutes. Anaesthesia with these quantities lasted from fourteen to twenty minutes, when, if the operation required it, open $\mathrm{E}_{2} \mathrm{C}_{1}$ followed. 